\title{
rAAV-mediated overexpression of TGF- $\beta$ via vector delivery in polymeric micelles stimulates the biological and reparative activities of human articular chondrocytes in vitro and in a human osteochondral defect model
}

This article was published in the following Dove Press journal:

International Journal of Nanomedicine

19 September 2017

Number of times this article has been viewed

\author{
Ana Rey-Rico' \\ Jagadeesh K Venkatesan' \\ Gertrud Schmitt' \\ Angel Concheiro ${ }^{2}$ \\ Henning Madry 1,3 \\ Carmen Alvarez-Lorenzo ${ }^{2}$ \\ Magali Cucchiarini' \\ 'Center of Experimental Orthopedics, \\ Saarland University Medical Center, \\ Homburg, Germany; ' ${ }^{2}$ epartment \\ of Pharmacology, Pharmacy \\ and Pharmaceutical Technology, \\ R+ DPharma Group (GI-I645), \\ Facultad de Farmacia and Health \\ Research Institute of Santiago de \\ Compostela (IDIS), Universidade de \\ Santiago de Compostela, Santiago de \\ Compostela, Spain; ${ }^{3}$ Department of \\ Orthopedics and Orthopedic Surgery, \\ Saarland University Medical Center, \\ Homburg, Germany
}

\begin{abstract}
Recombinant adeno-associated virus (rAAV) vectors are clinically adapted vectors to durably treat human osteoarthritis (OA). Controlled delivery of rAAV vectors via polymeric micelles was reported to enhance the temporal and spatial presentation of the vectors into their targets. Here, we tested the feasibility of delivering rAAV vectors via poly (ethylene oxide) (PEO) and poly (propylene oxide) (PPO) (poloxamer and poloxamine) polymeric micelles as a means to overexpress the therapeutic factor transforming growth factor-beta (TGF- $\beta$ ) in human OA chondrocytes and in experimental human osteochondral defects. Application of rAAV-human transforming growth factor-beta using such micelles increased the levels of TGF- $\beta$ transgene expression compared with free vector treatment. Overexpression of TGF- $\beta$ with these systems resulted in higher proteoglycan deposition and increased cell numbers in OA chondrocytes. In osteochondral defect cultures, a higher deposition of type-II collagen and reduced hypertrophic events were noted. Delivery of therapeutic rAAV vectors via PEO-PPOPEO micelles may provide potential tools to remodel human OA cartilage.
\end{abstract}

Keywords: controlled delivery, human articular cartilage, rAAV gene transfer, TGF- $\beta$, poloxamer, poloxamine

\section{Introduction}

Osteoarthritis (OA) is a major, chronic joint disorder that involves deep changes in the structure and functions of skeletal tissues and cells, causing the gradual and irreversible breakdown of the articular cartilage (the end point of OA), the remodeling of the subchondral bone, and the formation of osteophytes ${ }^{1,2}$ by an impaired homeostasis..$^{3,4}$ Current pharmacological and surgical strategies cannot fully reverse the OA phenotype and the original cartilage integrity is never being recovered. The design of new, durable, and effective approaches is thus under active investigation to avoid the progression of tissue degeneration while stably repairing the lesions. Gene therapy is a valuable strategy to achieve this goal, allowing to directly transfer genes coding for candidate factors within the repair tissue as a means to prolong their therapeutic activities. ${ }^{5,6}$

Protection against OA cartilage breakdown via gene transfer has been reported by applying sequences for agents displaying preventive and/or inhibitory activities (IL-1 receptor antagonist, inhibitors of nuclear factor- $\kappa \mathrm{B}$, thrombospondin-1, kallistatin, 
pro-opiomelanocortin, Dickkopf-1). ${ }^{7-14}$ In parallel, activation of anabolic/proliferative processes in OA has also been attempted using therapeutic genes such as the insulin-like growth factor $\mathrm{I},{ }^{15,16}$ basic fibroblast growth factor-2, ${ }^{17}$ bone morphogenetic proteins, ${ }^{18}$ the transcription factor SOX9, ${ }^{19}$ and proteoglycan $4 .{ }^{20}$ The transforming growth factor-beta (TGF- $\beta$ ) is another highly potent agent capable of promoting cartilage repair, enhancing the proliferation of chondrocytes and production of extracellular cartilage matrix (ECM) components and preventing cartilage degradation. ${ }^{21-23}$ While gene transfer and overexpression of TGF- $\beta$ was evaluated in articular chondrocytes in vitro and in situ via nonvira ${ }^{24,25}$ and classical viral (adenoviruses and retroviruses) vectors, ${ }^{26-28}$ the use of such vehicles in vivo may be impaired by their low and short-term efficiencies, especially in nondividing cells like chondrocytes, by the possibility of insertional mutagenesis (retroviral vectors), and their relatively high immunogenicity (adenoviral vectors). ${ }^{5,6}$ In marked contrast, recombinant vectors based on the human adeno-associated virus (AAV) exhibit high, extended gene transfer efficiencies in chondrocytes in vitro and in situ within their dense ECM (up to $80 \%$ for at least 150 days) ${ }^{29}$ due to their small size $(\sim 20 \mathrm{~nm})$ and their maintenance as stable episomes, making them particularly adapted for in vivo setups. ${ }^{30}$ Yet, while gene transfer of a recombinant adeno-associated virus (rAAV) TGF- $\beta$ construct allowed to stimulate the activity of chondrocytes in cartilage explants in situ, ${ }^{31,32}$ the performance of this vector in vivo may be hindered by neutralizing antibodies against the AAV capsid proteins mainly in the synovial fluid of patients with joint diseases..$^{33}$

Providing rAAV vectors via biocompatible materials as controlled delivery systems is a novel, potent approach to overcome such pre-existing barriers. ${ }^{34,35}$ In this regard, we previously reported that polymeric micelles that rely on poly (ethylene oxide) (PEO) and poly (propylene oxide) (PPO) copolymers in the form of linear poloxamers or X-shaped poloxamines may improve the stability and bioactivity of reporter (lacZ) rAAV gene vectors, especially those with a higher PEO/PPO ratio (poloxamer PF68 and poloxamine T908) ${ }^{36}$ In addition, encapsulation of rAAV in PF68 or T908 polymeric micelles resulted in an effective gene transfer of the reporter gene lac $Z$ in human OA chondrocytes in vitro and in experimental osteochondral defects without detrimental effects on the biological activities of the cells nor on their phenotype, also affording protection when anti-AAV capsid neutralizing antibodies were present. ${ }^{37}$ In light of such promising findings, the purpose of the present study was to test whether PF68 and T908 polymeric micelles can deliver a candidate rAAV TGF- $\beta$ vector to human OA chondrocytes and to human osteochondral defects in order to overexpress the growth factor as a potent therapeutic approach for the future treatment of articular cartilage injuries.

\section{Materials and methods Materials}

Pluronic ${ }^{\circledR}$ F68 and Tetronic ${ }^{\circledR} 908$ were kindly provided by BASF (Ludwigshafen, Germany). The anti-TGF- $\beta$ (V) was from Santa Cruz Biotechnology (Heidelberg, Germany). The anti-type-II collagen (II-II6B3) antibody was from DSHB (Iowa City, IA, USA) and the anti-type-X collagen (COL-10) antibody from Sigma (Munich, Germany). Biotinylated secondary antibodies and the $\mathrm{ABC}$ reagent were from Vector Laboratories (Alexis Deutschland $\mathrm{GmbH}$, Grünberg, Germany). The Cy3 Ab Labeling Kit was from Amersham/GE Healthcare (Munich, Germany). The cell proliferation reagent WST-1 and the Cytotoxicity Detection $\mathrm{Kit}^{\mathrm{PLUS}}$ (LDH) were from Roche Applied Science (Mannheim, Germany). The TGF- $\beta$ enzyme-linked immunosorbent assay (ELISA) (hTGF- $\beta$ Quantikine ELISA) was from R\&D Systems (Wiesbaden, Germany). Other reagents were from Sigma (Munich, Germany).

\section{Cells and osteochondral defect model}

Human OA cartilage (Mankin score of 7-9) was from total knee arthroplasty samples $(n=7)$ from patients who previously signed informed consent. ${ }^{32}$ The study was approved by the Ethics Committee of the Saarland Physicians Council (Approval Ha67/12) and all procedures were in accordance with the Helsinki Declaration.

Human OA chondrocytes were isolated as previously described $^{32}$ and used not later than passage 3. Cells were incubated at the denoted cell densities in DMEM, $10 \%$ fetal bovine serum, $100 \mathrm{U} / \mathrm{mL}$ penicillin $\mathrm{G}, 100 \mu \mathrm{L} / \mathrm{mL}$ streptomycin (growth medium) for $12 \mathrm{~h}$ at $37^{\circ} \mathrm{C}$ under $5 \%$ $\mathrm{CO}_{2}$ prior to addition of the $\mathrm{rAAV} /$ copolymer systems or free rAAV preparations (see below for concentrations) for up to 10 days for consistency with our previous study with reporter vectors. ${ }^{37}$

Osteochondral defects were created in human OA cartilage biopsies $(\mathrm{n}=7)$ using a 1-mm drill needle in standardized cylindrical (6-mm diameter) as previously described ${ }^{37}$ and incubated in growth medium prior to addition of the rAAV/ copolymer systems or free rAAV preparations at the concentrations indicated thereafter for 10 days.

\section{Plasmids and rAAV vectors}

The constructs were derived from pSSV9, an AAV-2 genomic clone. ${ }^{38,39}$ rAAV-hTGF- $\beta$ carries a 1.2 -kb human 
transforming growth factor-beta 1 (hTGF- $\beta$ ) cDNA fragment under the control of the cytomegalovirus immediate-early promoter. ${ }^{32,36,40}$ The vectors were packaged as conventional (not self-complementary) vectors using a helper-free, 2-plasmid transfection system in 293 cells with the packaging plasmid pXX2 and the adenovirus helper plasmid pXX6. ${ }^{32}$ The vector preparations were purified by extensive dialysis and titrated by real-time polymerase chain reaction, ${ }^{32,36,40}$ averaging $10^{10}$ transgene copies/mL ( 1/500 functional recombinant viral particles).

\section{Cy3 labeling}

rAAV vectors were labeled using the $\mathrm{Cy} 3 \mathrm{Ab}$ Labeling Kit as previously described. ${ }^{41}$ Briefly, rAAV (1 mL) was dispersed in sodium carbonate/sodium bicarbonate buffer $(\mathrm{pH} 9.3)$, kept for $30 \mathrm{~min}$ at $20^{\circ} \mathrm{C}$, and purified by extensive dialysis against $20 \mathrm{mM}$ HEPES ( $\mathrm{pH} 7.5$ )/150 mL NaCl. Effective labeling was monitored in the samples by live fluorescent microscopy with rhodamine filter set (Olympus CKX41; Hamburg, Germany).

\section{Preparation of copolymer solutions containing rAAV vectors}

The appropriate amounts of each type of copolymer (PF68 or T908) were added to a given volume of $10 \%$ sucrose aqueous solution at $4{ }^{\circ} \mathrm{C}$. The obtained poloxamer and poloxamine solutions were then mixed with rAAV (or Cy3-labeled rAAV; $10^{10}$ transgene copies $/ \mathrm{mL}$ ) in equal volumes, kept in ice-water bath for $30 \mathrm{~min}$, and used. ${ }^{36}$ The final copolymer concentration into the medium was always $2 \%$. Characterization of the systems revealed an effective association between the vectors and the polymeric micelles, also supported by the increase in size of the aggregates as recorded by transmission electron microscopy and dynamic light scattering. ${ }^{37}$

\section{Gene transfer efficacy using the rAAV/ copolymer systems}

Monolayer cultures of human OA chondrocytes (3,000 cells/ well in 96-well plates) and osteochondral defect cultures were directly incubated with the $\mathrm{rAAV} / \mathrm{PF} 68$ or $\mathrm{rAAV} / \mathrm{T} 908$ micelles (20 or $40 \mu \mathrm{L}$ for monolayer cultures; $100 \mu \mathrm{L}$ for osteochondral defect cultures; final copolymer concentration $2 \%$ ) and kept for 10 days at $37^{\circ} \mathrm{C}$ with 3 weekly medium change. ${ }^{36,40}$ Control conditions included application of $10 \%$ sucrose aq solution (negative control), similar amounts of free rAAV (10 or $20 \mu \mathrm{L}$ for monolayer cultures; $40 \mu \mathrm{L}$ for osteochondral defect cultures; $10^{10}$ transgene copies $/ \mathrm{mL}$ ) in $10 \%$ sucrose (v/v; positive control), and copolymer with $10 \%$ sucrose (w/v; copolymer control). Application of rAAV carrying a reporter (lacZ) gene was not included in this study due to its absence of effects on chondrocytes metabolic activities in accordance with our previous studies. ${ }^{36,42}$

\section{Detection of transgene expression}

Expression of TGF- $\beta$ was assayed by ELISA. ${ }^{32,39}$ Briefly, samples (cells in monolayer culture, osteochondral defect cultures) were washed twice and maintained for $24 \mathrm{~h}$ in serum-free medium previously to the collection of the culture medium supernatants. At given time intervals, supernatants were taken and centrifuged to separate debris. Measurements were carried out on a GENios apparatus (Tecan, Crailsheim, Germany). Additionally, TGF- $\beta$ expression was quantified by immunocyto/histochemistry using a specific primary antibody, a biotinylated secondary antibody, and the ABC method with diaminobenzidine as the chromogen. ${ }^{32,39}$ Secondary immunoglobulins were monitored in sections processed without addition of the primary antibody and then visualized using a light microscope (Olympus BX 45).

\section{Assessment of cell proliferation}

Proliferation of cells in monolayer culture was quantified with the cell proliferation reagent WST-1. ${ }^{36,40}$ Controls included cells maintained with or without same dose of free vector, and also with copolymer in the absence of rAAV.

\section{Assessment of cell viability}

Viability of cells in monolayer culture and in osteochondral defect cultures was determined with the Cytotoxicity Detection $\mathrm{Kit}^{\text {PLUS }}$ (LDH; Roche Applied Science, Mannheim, Germany) in the supernatants of culture by recording the absorbance at $450 \mathrm{~nm}$ (GENios, Tecan). ${ }^{37,42}$ Cytotoxicity was calculated as follows:

$$
\begin{aligned}
& \text { Cytotoxicity }(\%) \\
& \qquad=\left(\frac{\text { Experimental value }- \text { Low control }}{\text { High control }- \text { Low control }}\right) \times 100
\end{aligned}
$$

where low control corresponds to samples without assay treatment and high control to samples placed in the lysis buffer provided by the kit.

\section{Histological and immunocyto/ histochemical analyses}

Cells in monolayer culture and osteochondral defect cultures were harvested at the denoted time points and fixed in $4 \%$ formalin. ${ }^{37}$ Fixed cells were stained with Alcian blue (matrix proteoglycans) and excess stain was washed off with 
double distilled water. The stain was quantified by overnight solubilization in $6 \mathrm{M}$ guanidine hydrochloride to monitor absorbance at $595 \mathrm{~nm}$ (GENios, Tecan)..$^{37,43,44}$ Fixed osteochondral defect cultures were further dehydrated in graded alcohols, embedded in paraffin, and sectioned $(10 \mu \mathrm{m})$. Sections were stained with H\&E (cellularity), safranin O (matrix proteoglycans), and alizarin red (matrix mineralization) as previously described. ${ }^{32,36,40}$ Expression of TGF- $\beta$ and type-II/-X collagen was monitored by immunocyto/ histochemistry as indicated previously..$^{32,36,40}$ To control for secondary immunoglobulins, sections were processed with omission of the primary antibody.

\section{Histomorphometry}

The cell densities (cell number $/ \mathrm{mm}^{2}$ ) in H\&E-stained sections, and the intensities of safranin O, type-II collagen and alizarin red staining and those of TGF- $\beta$ and type-X collagen immunostaining (ratio of tissue surface positively stained to the total surface of the site evaluated on histological sections) were measured at four randomized sites as explained elsewhere using SIS AnalySIS (Olympus) and Adobe Photoshop Adobe Systems software (Unterschleissheim, Germany). ${ }^{32,36,40}$

\section{Statistical analysis}

Three independent experiments were carried out for each condition, which was evaluated in duplicate. Data are expressed as mean \pm SD. Statistical analysis was performed using SPSS version 23 (IBM, Armonk, NY, USA) and a $p$-value $\leq 0.05$ was considered statistically significant. One-way analysis of variance with Tukey's LSD or Games-Howell post hoc tests was applied to evaluate differences between groups.

\section{Results}

\section{Efficacy of rAAV-mediated TGF- $\beta$ overexpression in human $O A$ chondrocytes via vector delivery using PEO-PPO-PEO copolymers}

We first tested whether the PEO-PPO-PEO copolymers were capable of successfully delivering the rAAV-hTGF- $\beta$ candidate vector to human OA chondrocytes in monolayer culture in vitro.

A quantitative estimation of TGF- $\beta$ production revealed efficient levels of transgene expression in the cells upon rAAV-mediated gene delivery via the polymeric micelles (Figure 1A), with significant, up to 2.7-fold difference relative to free vector treatment $(p \leq 0.040)$ and up to 19.5-fold difference versus control condition $(p \leq 0.020)$. Furthermore, significantly increased levels of expression were seen over time with PF68 at the highest vector dose and T908 at either dose applied (up to 1.4-fold increase between days 3 and 10, $p \leq 0.020$ ). A significant vector dose-dependent effect was also observed when using micelles, especially after 10 days of treatment (up to 2.1-fold difference between 10 and $20 \mu \mathrm{L}, p \leq 0.050$ ). Similar trends were observed when TGF- $\beta$ expression was monitored by immunocytochemical analysis (up to 1.4-fold increase relative to free vector treatment, $p=0.010$; up to 20.7-fold difference versus control condition, $p \leq 0.002$ ) (Figure 1B and C), with a significant increase in transgene expression over time (up to 1.3-fold difference between days 3 and 10, $\leq \leq 0.010$ ) and a significantly more intense TGF- $\beta$ immunoreactivity at the highest vector dose applied (up to 1.7-fold difference between 10 and $20 \mu \mathrm{L}$ on day $10, p=0.010)$.

\section{Effects of rAAV-hTGF- $\beta$ /PEO-PPO-PEO copolymer application on the biological activities of human OA chondrocytes}

We next examined the potentially therapeutic effects of TGF- $\beta$ overexpression upon delivery of rAAV-hTGF- $\beta$ via PEO-PPO-PEO copolymers in human OA chondrocytes in vitro.

Analysis of the proliferative activities in the cells revealed durably increased indices following rAAV-hTGF- $\beta$-mediated gene transfer (up to 1.8-fold increase compared with the control, $p \leq 0.010$ ), especially when delivering the vectors via the polymeric micelles, although statistically significant difference was not reached (up to 1.4-fold increase versus free vector treatment, $p \leq 0.210$ ) (Figure 2A). A vector dosedependent effect on cell proliferation was observed early on, although statistically significant differences were not reached (up to 1.3-fold increase between 10 and $20 \mu \mathrm{L} ; p \leq 0.290$ ). So far, this effect was not observed after 10 days, showing a fold-decrease with increased vector doses (up to 0.7-fold decrease via delivery of AAV from T908 polymeric micelles; $p \leq 0.040$ ). Equally important, no deleterious effects of any of the gene transfer approaches (polymeric micelles, free vector treatment) were reported at any time point and regardless of the vector dose employed relative to the control condition ( $p \geq 0.090)$ (Figure 2B).

Finally, rAAV-mediated TGF- $\beta$ overexpression promoted a significantly sustained deposition of a proteoglycan-rich ECM in treated cells to levels that were higher than those noted in the control conditions, especially upon delivery via the polymeric micelles (up to 1.5-fold difference, $p \leq 0.0001$ ) (Figure 2C and D). Besides, a vector dose dependence on ECM deposition was noted (up to 1.2-fold difference between 10 and $20 \mu \mathrm{L} ; p \leq 0.010)$. 


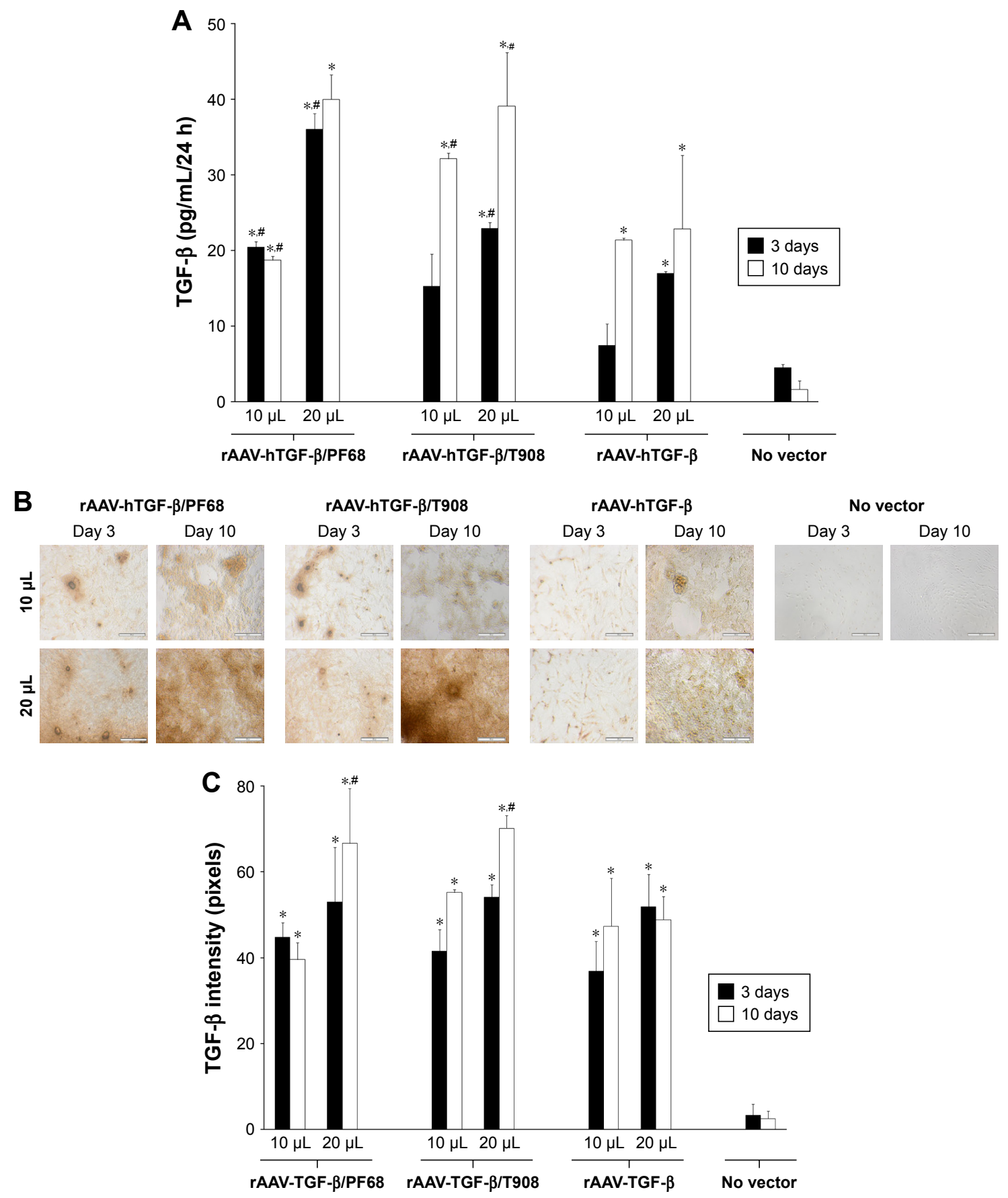

Figure I Transgene expression in rAAV-hTGF- $\beta$-transduced human OA chondrocytes in the presence of polymeric micelles.

Notes: Cells in monolayer culture were prepared and incubated with rAAV/polymeric micelles and the cultures were processed at the denoted time points to monitor TGF- $\beta$ expression (A) by enzyme-linked immunosorbent assay and (B) by immunohistochemical detection (magnification $\times 4$, all representative data; scale bar $500 \mu \mathrm{m}$ ) with (C) corresponding histomorphometric analyses. Control conditions included the absence of copolymer or vector treatment (negative control) and application of free rAAV vector (positive control). Statistically significant compared with *negative and "positive controls.

Abbreviations: hTGF, human transforming growth factor; OA, osteoarthritis; rAAV, recombinant adeno-associated virus; TGF- $\beta$, transforming growth factor-beta.

Efficacy of rAAV-mediated TGF- $\beta$ overexpression in human osteochondral defect cultures via gene vector delivery using PEO-PPO-PEO copolymers

We then evaluated whether the PEO-PPO-PEO copolymers were capable of successfully delivering the rAAV-hTGF- $\beta$ candidate vector to human osteochondral defect cultures in situ.

Application of Cy3-labeled rAAV-hTGF- $\beta$ revealed a diffusion of bioactive rAAV vectors in-depth within the defects when administered in both free form or encapsulated in PF68 and T908 micelles in an early on setup (Figure S1). 
A

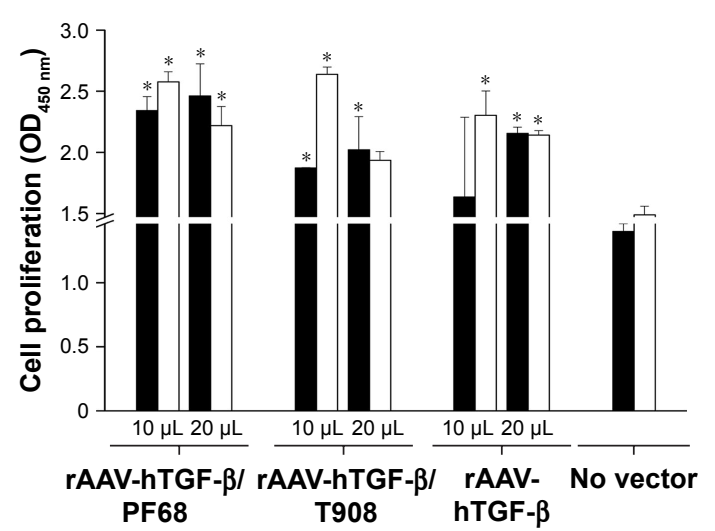

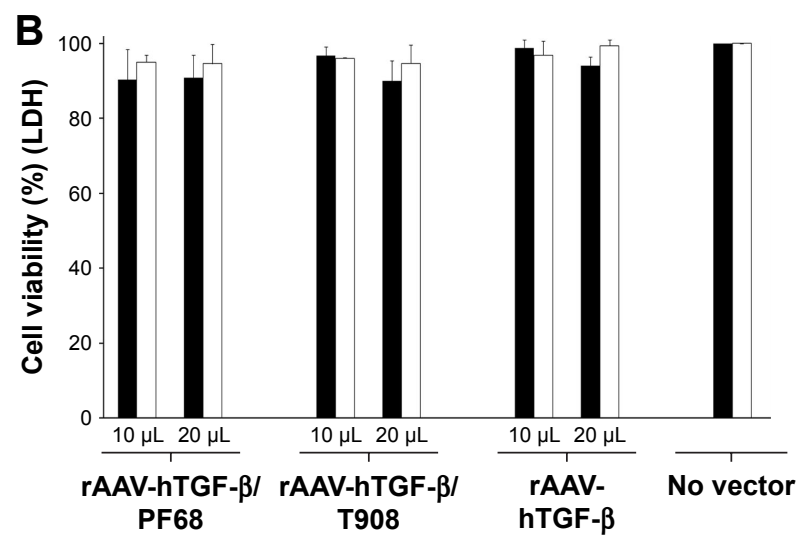

C

rAAV-hTGF- $\beta /$ PF68

Day 3

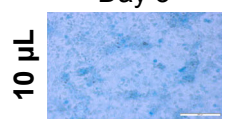

ำ 살
Day 10
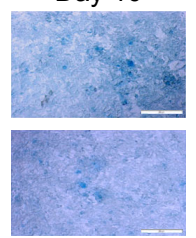

rAAV-hTGF- $\beta$ /T908
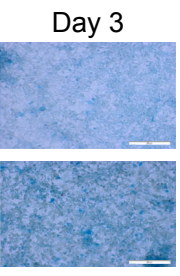

Day 10

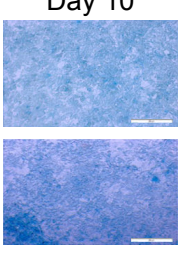

rAAV-hTGF- $\boldsymbol{\beta}$

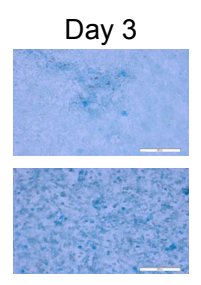

No vector
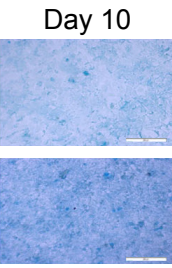
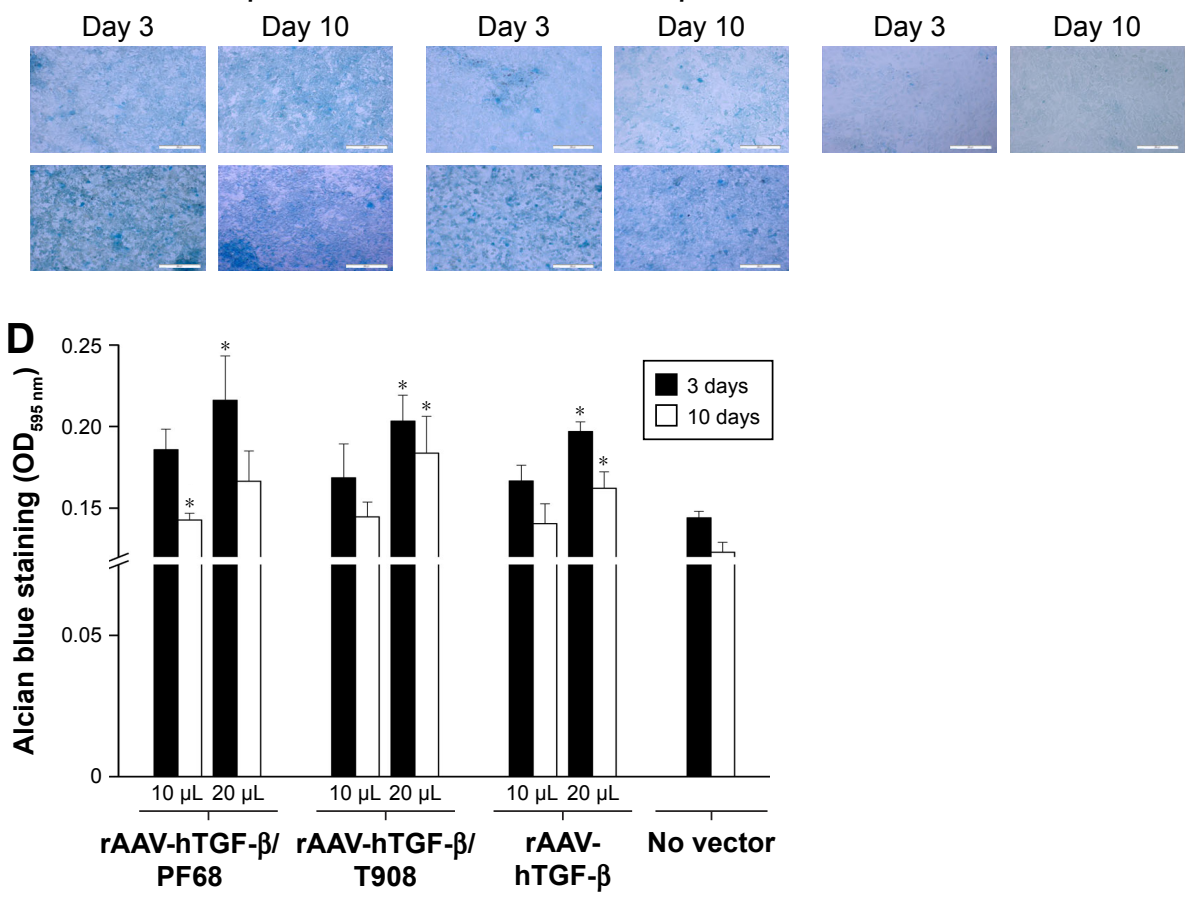

Figure 2 Biosynthetic activities in rAAV-hTGF- $\beta$-transduced human OA chondrocytes in the presence of polymeric micelles.

Notes: Cells in monolayer culture were prepared and incubated with rAAV/polymeric micelles and the cultures were processed at the denoted time points to monitor cell viability by WST-I assay (A), LDH assay (B), matrix production (C), and by Alcian blue staining (magnification $\times 10$, all representative data; scale bar $200 \mu \mathrm{m})(\mathbf{D})$, with spectrophotometric analyses after solubilization in $6 \mathrm{M}$ guanidine hydrochloride. Control conditions included the absence of copolymer or vector treatment (negative control) and application of free rAAV vector (positive control). *Statistically significant compared with negative control.

Abbreviations: hTGF, human transforming growth factor; LDH, lactate dehydrogenase; OA, osteoarthritis; $r A A V$, recombinant adeno-associated virus; TGF- $\beta$, transforming growth factor-beta.

As observed for monolayer cultures of human OA chondrocytes, efficient TGF- $\beta$ production was noted in defect cultures upon rAAV-hTGF- $\beta$-mediated gene transfer for at least 10 days, especially when applying the vectors via PF68-based micelles (up to 1.6-fold difference relative to the control condition), even though statistically significant differences were not reached ( $p \leq 0.333$ ) (Figure 3A). Similar trends were observed when TGF- $\beta$ expression was monitored by immunohistochemical analysis (up to 14.5 -fold relative to control condition, $p \leq 0.0001$ ) (Figure $3 \mathrm{~B}$ and $\mathrm{C}$ ). A higher TGF- $\beta$ immunoreactivity was noted by applying rAAV either via T908-based micelles or in its free form compared with their administration via T908-based micelles $(p \leq 0.035)$.

\section{Effects of rAAV-hTGF- $\beta / P E O-P P O-$}

\section{PEO copolymer application in human} osteochondral defect cultures

We finally examined the potential effects of TGF- $\beta$ overexpression in human osteochondral defect cultures upon delivery of rAAV-hTGF- $\beta$ via PEO-PPO-PEO copolymers.

Cell densities in regions adjacent to the defects were significantly higher upon rAAV-hTGF- $\beta$-mediated gene transfer 

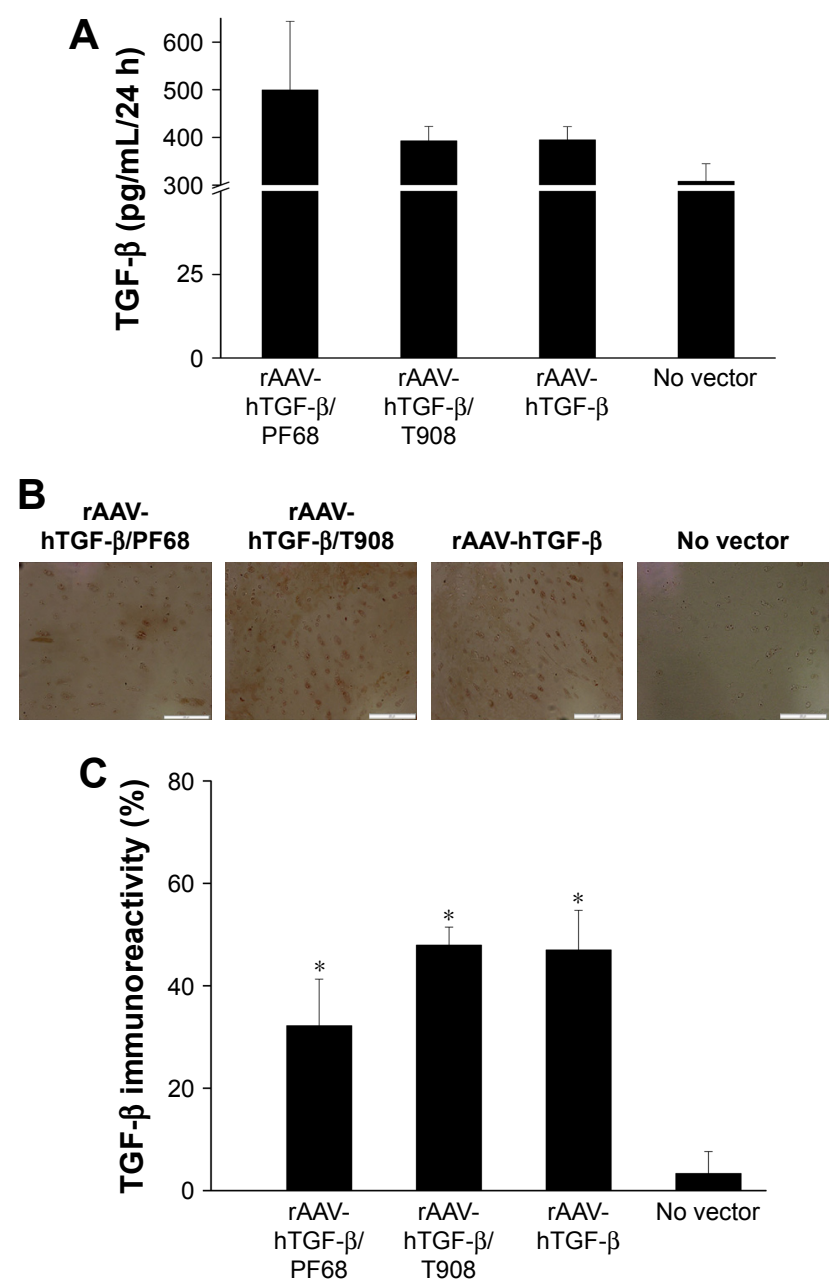

Figure 3 Transgene expression in rAAV-hTGF- $\beta$-transduced human osteochondral defect cultures in the presence of polymeric micelles.

Notes: Defects were prepared and incubated with rAAV/polymeric micelles and the cultures were processed after 10 days to monitor TGF- $\beta$ expression (A), enzyme-linked immunosorbent assay (B), and by immunohistochemical detection (magnification $\times 10$, all representative data; scale bar $200 \mu \mathrm{m})(\mathbf{C})$, with corresponding histomorphometric analyses. Control conditions included the absence of copolymer or vector treatment (negative control) and application of free $\mathrm{rAAV}$ vector (positive control). Statistically significant compared with *negative controls.

Abbreviations: hTGF, human transforming growth factor; rAAV, recombinant adeno-associated virus; TGF- $\beta$, transforming growth factor-beta.

(up to 2.3-fold difference versus control condition, $p \leq 0.050$ ) (Figure 4A and B). Here also, no deleterious effects were observed in the cultures, independently from the delivery system tested (polymeric micelles and free vector treatment) relative to the control condition ( $p \geq 0.342)$ (Figure 4C).

In addition, $\mathrm{rAAV}$-mediated TGF- $\beta$ overexpression significantly enhanced the production of proteoglycan-rich ECM in treated defects with respect to the controls (up to 1.2-fold difference, $p=0.010$ ), especially upon delivery via T908 micelles ( $p=0.036$ ) (Figure 5A and B). Administration of rAAV-hTGF- $\beta$ also led to significantly higher levels of type-II collagen deposition relative to the control condition (up to 1.3 -fold difference, $p=0.0001$ ), especially upon
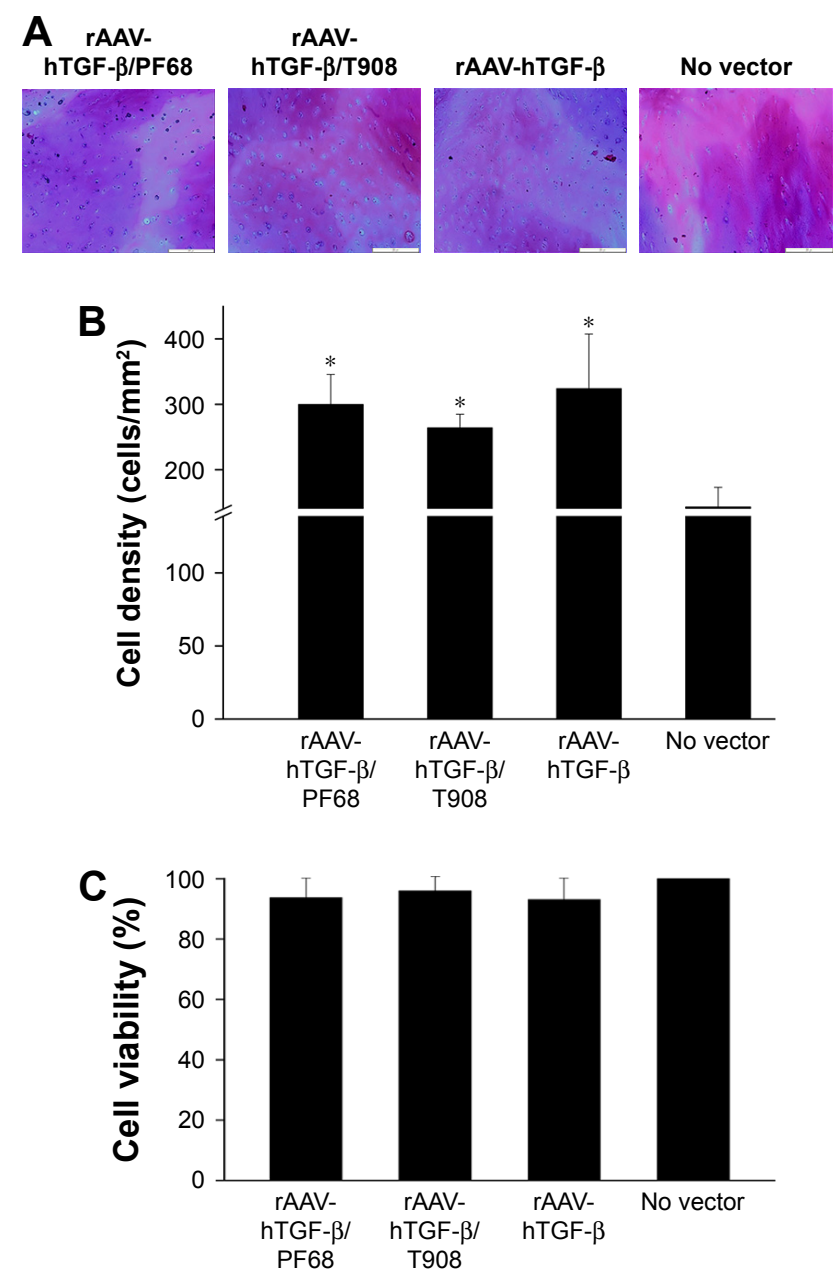

Figure 4 Cell viability in rAAV-hTGF- $\beta$-transduced human osteochondral defect cultures in the presence of polymeric micelles.

Notes: Defects were prepared and incubated with rAAV/polymeric micelles and the cultures were processed after 10 days for $($ A) H\&E staining (magnification $\times 10$, all representative data; scale bar $200 \mu \mathrm{m}$ ) with (B) corresponding histomorphometric analyses and (C) to monitor cell viability using the LDH assay. Control conditions included the absence of copolymer or vector treatment (negative control) and application of free rAAV vector (positive control). *Statistically significant compared with negative control.

Abbreviations: hTGF, human transforming growth factor; LDH, lactate dehydrogenase; rAAV, recombinant adeno-associated virus.

delivery via PF68 micelles $(p \leq 0.010)$ (Figure 5C and D). Nevertheless, in both cases, the levels achieved upon delivery via the polymeric micelles were lower than those promoted upon free vector treatment at this time point and vector dose $(p \leq 0.010)$. Most strikingly, an estimation of type-X collagen expression in regions adjacent to the defects revealed a significant reduction of undesirable cartilage hypertrophy upon rAAV-hTGF- $\beta$-mediated transduction (up to 28.8fold difference versus control condition, $p \leq 0.040$ ) while no differences were observed in all groups treated with rAAV-hTGF- $\beta$ ( $p \geq 0.783$ ) (Figure 6A and B). In addition, an analysis of alizarin red staining intensity in regions adjacent to the defects showed a significant reduction of matrix 


\section{A}
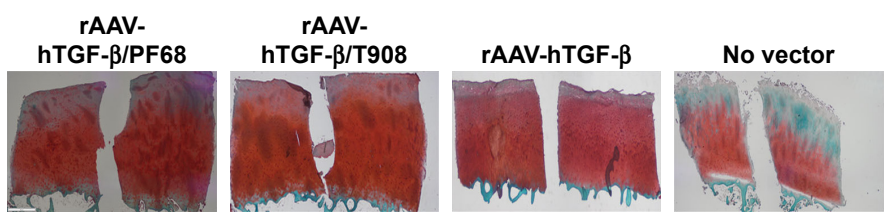

C
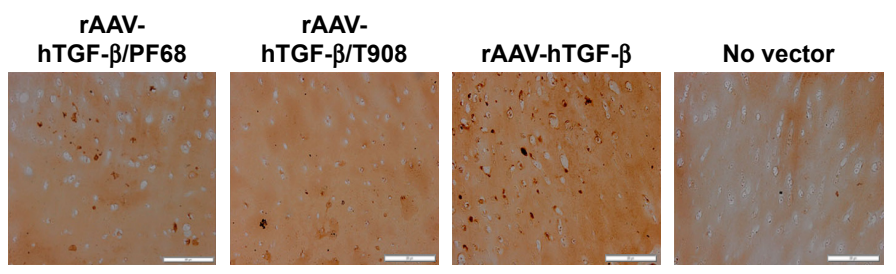
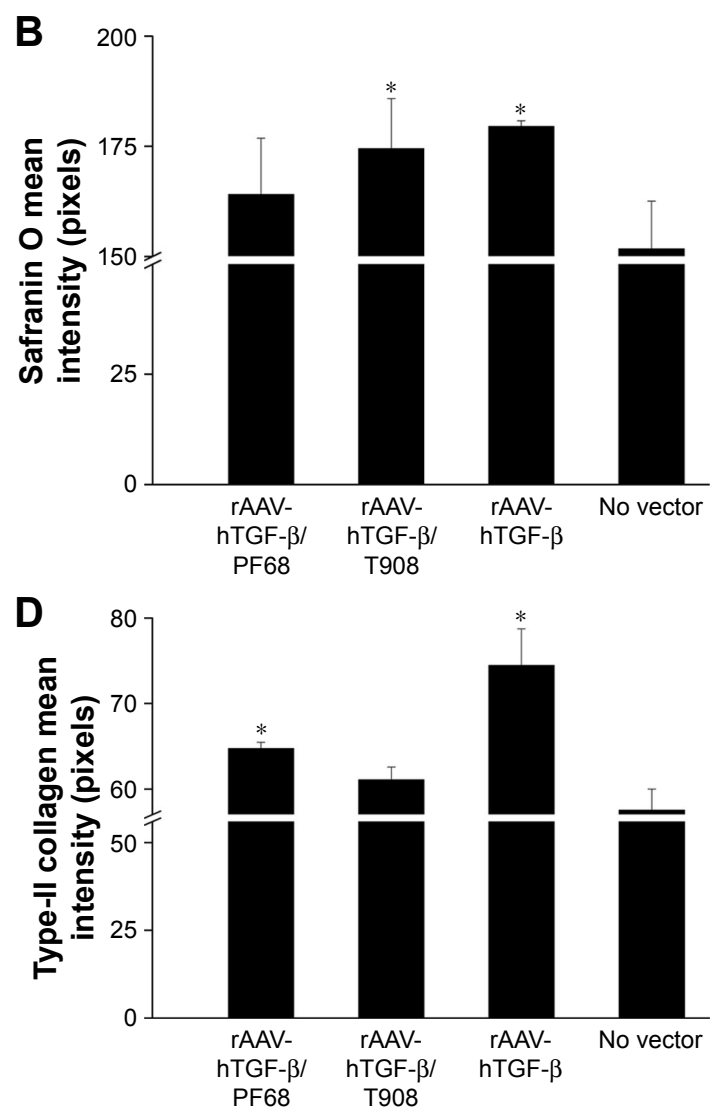

Figure 5 Biosynthetic activities in rAAV-hTGF- $\beta$-transduced human osteochondral defect cultures in the presence of polymeric micelles.

Notes: Defects were prepared and incubated with rAAV/polymeric micelles and the cultures were processed after 10 days for $(\mathbf{A})$ safranin $O$ staining and (C) for immunohistochemical detection of type-Il collagen (magnification $\times 10$, all representative data; scale bar $200 \mu \mathrm{m}$ ) with (B, D) corresponding histomorphometric analyses. Control conditions included the absence of copolymer or vector treatment (negative control) and application of free rAAV vector (positive control). *Statistically significant compared with negative control.

Abbreviations: hTGF, human transforming growth factor; rAAV, recombinant adeno-associated virus.

mineralization upon rAAV-hTGF- $\beta$-mediated gene transfer (up to 1.2-fold difference versus control condition, $p \leq 0.047$ ) (Figure 6C and D). No differences were observed between groups treated with rAAV-hTGF- $\beta$, independently from the delivery system tested (polymeric micelles, free vector treatment) ( $p \geq 0.939)$.

\section{Discussion}

Gene transfer based on $\mathrm{rAAV}$ vectors represents a valuable tool to manage a gradual disease like OA as these constructs allow for a durable repair of damaged articular cartilage in situ $^{16,29,32}$ versus other less effective gene carriers (nonviral, adenoviral, and retroviral vectors). ${ }^{5,6}$ Yet, the clinical application of rAAV is precluded by the natural presence of neutralizing antibodies in the patients, especially by those against the viral capsid proteins. ${ }^{45}$ Delivery of rAAV via PEO-PPOPEO polymeric micelles is a potent approach to overcome these hurdles that impair the efficacy of rAAV-mediated gene transfer. ${ }^{36,37}$ We previously reported that delivery of
rAAV carrying the reporter gene lac $Z$ via polymeric micelles increased both the stability and bioactivity of rAAV vectors, promoting higher levels of safe transgene in vitro and in experimental osteochondral defect models. ${ }^{37}$ Here, based on such observations, we tested the therapeutic benefits of providing rAAV vectors overexpressing the potent factor TGF- $\beta$ to human OA chondrocytes using PEO-PPO-PEObased polymeric micelles.

The data first indicate that administration of rAAVhTGF- $\beta$ via polymeric micelles to human OA chondrocytes increased up to 2.7-fold the levels of TGF- $\beta$ transgene expression over time compared with free vector treatment. Beneficial effects of rAAV-mediated TGF- $\beta$ overexpression in human OA chondrocytes were confirmed by increased indices of cell proliferation, especially after administration of the vector via polymeric micelles. Of note and in accordance with previous studies using similar systems, ${ }^{36,37}$ there was no detrimental effect of the treatments on cell viability over the whole test period using either encapsulated vectors or free 


\section{A}

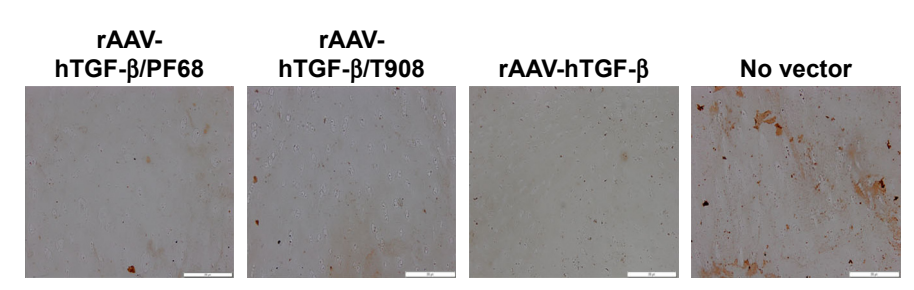

C

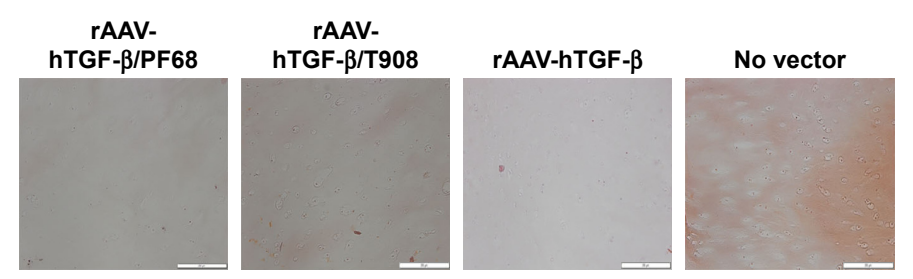

B

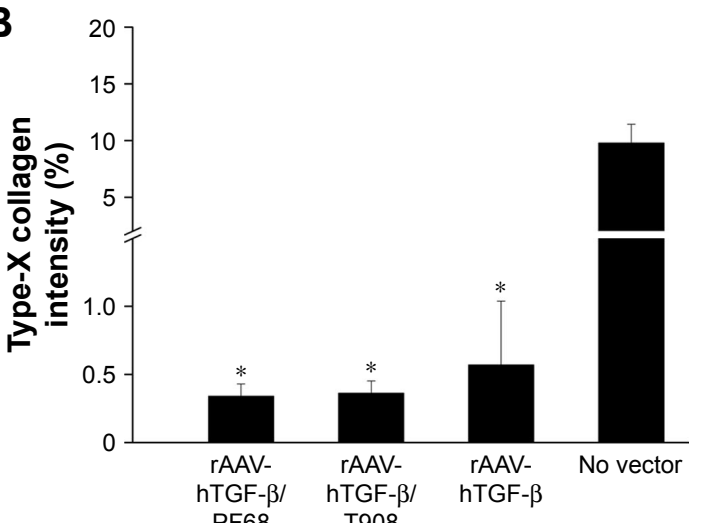

D

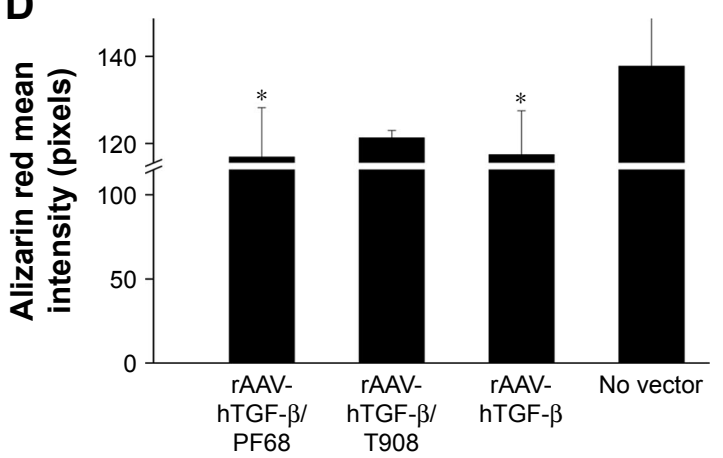

Figure 6 Hypertrophic and terminal differentiation activities in rAAV-hTGF- $\beta$-transduced human osteochondral defect cultures in the presence of polymeric micelles. Notes: Defects were prepared and incubated with rAAV/polymeric micelles and the cultures were processed after 10 days for immunohistochemical detection of (A) type-X collagen and (C) alizarin red staining (all at magnification $\times 10$, all representative data; scale bar $200 \mu \mathrm{m}$ ) with (B, D) corresponding histomorphometric analyses. Control conditions included the absence of copolymer or vector treatment (negative control) and application of free rAAV vector (positive control). *Statistically significant compared with negative control.

Abbreviations: hTGF, human transforming growth factor; rAAV, recombinant adeno-associated virus.

vector preparations. Overexpression of TGF- $\beta$ in human OA chondrocytes resulted in higher proteoglycan-rich ECM deposition relative to the untreated control groups, especially upon administration of the vector via polymeric micelles.

The results further show the suitability of the current approach to modify cells in a more natural, translational environment by providing therapeutic rAAV-hTGF- $\beta$ via PEO-PPO-PEO micelles to a human osteochondral defect model. ${ }^{37}$ Quantitative estimation of the cell densities in regions adjacent to the defects revealed increased cell numbers upon rAAV-hTGF- $\beta$ transduction independently from the method of delivery applied (polymeric micelles, free vector treatment), consistent with the effects of TGF- $\beta$ in human normal and OA cartilage explant cultures. ${ }^{32}$ Furthermore, administration of rAAV-hTGF- $\beta$ either via polymeric micelles or in its free form increased the proteoglycans contents in human osteochondral defect cultures. Similarly, overexpression of TGF- $\beta$ in human osteochondral defect cultures resulted in higher deposition of type-II collagen compared with the control groups ${ }^{46}$ These results highlight the role that rAAV-mediated TGF- $\beta$ gene transfer may play in remodeling human OA cartilage by triggering biological and reparative activities. ${ }^{32}$ Interestingly, delivery of rAAV-hTGF- $\beta$ via polymeric micelles resulted in lower levels of type-II collagen compared with free vector treatment, at least at the time point and $\mathrm{rAAV} /$ copolymer ratio selected here.$^{37}$ Study is ongoing to extend the evaluation at longer time points and also using different $\mathrm{rAAV} /$ copolymer ratios in order to examine whether overexpression of TGF- $\beta$ from these systems may become more effective than when using free vector administration. Finally, and in accordance with our previous findings with human OA cartilage explants, ${ }^{32}$ a favorable reduction in hypertrophic events was observed upon rAAV-hTGF- $\beta$-mediated gene transfer of human osteochondral defect cultures.

Overall, the present study reveals the potential of using controlled delivery systems for rAAV-mediated TGF- $\beta$ gene transfer to improve the current gene-based treatments for human OA by increasing the temporal and spatial presentation of the gene products into the targets. Study is also ongoing to examine the potency of the approach in relevant animal models of OA in vivo. ${ }^{7-14,20}$ In conclusion, combining 
controlled delivery strategies via PEO-PPO-PEO micelles and therapeutic rAAV gene transfer may constitute powerful tools to remodel human OA cartilage.

\section{Acknowledgments}

This work was supported by a grant from Deutsche Forschungsgemeinschaft (DFG RE 328/2-1 to ARR, HM, MC), MINECO (SAF2014-52632-R) Spain, and FEDER. The authors thank RJ Samulski (The Gene Therapy Center, University of North Carolina, Chapel Hill, NC, USA), X Xiao (The Gene Therapy Center, University of Pittsburgh, Pittsburgh, PA, USA), and EF Terwilliger (Division of Experimental Medicine, Harvard Institutes of Medicine and Beth Israel Deaconess Medical Center, Boston, MA, USA) for providing the genomic AAV-2 plasmid clones, the pXX2 and pXX6 plasmids, and the 293 cell line.

\section{Disclosure}

The authors report no conflicts of interest in this work.

\section{References}

1. Loeser RF, Goldring SR, Scanzello CR, Goldring MB. Osteoarthritis: a disease of the joint as an organ. Arthritis Rheum. 2012;64(6): 1697-1707.

2. Poole AR. Osteoarthritis as a whole joint disease. HSS J. 2012;8(1):4-6.

3. Kapoor M, Martel-Pelletier J, Lajeunesse D, Pelletier JP, Fahmi H. Role of proinflammatory cytokines in the pathophysiology of osteoarthritis. Nat Rev Rheumatol. 2011;7(1):33-42.

4. Goldring MB, Otero M. Inflammation in osteoarthritis. Curr Opin Rheumatol. 2011;23(5):471-478.

5. Madry H, Cucchiarini M. Advances and challenges in gene-based approaches for osteoarthritis. J Gene Med. 2013;15(10):343-355.

6. Evans $\mathrm{CH}$, Huard J. Gene therapy approaches to regenerating the musculoskeletal system. Nat Rev Rheumatol. 2015;11(4):234-242.

7. Pelletier JP, Caron JP, Evans C, et al. In vivo suppression of early experimental osteoarthritis by interleukin-1 receptor antagonist using gene therapy. Arthritis Rheum. 1997;40(6):1012-1019.

8. Frisbie DD, Ghivizzani SC, Robbins PD, Evans CH, McIlwraith CW. Treatment of experimental equine osteoarthritis by in vivo delivery of the equine interleukin-1 receptor antagonist gene. Gene Ther. 2002; 9(1):12-20.

9. Chen LX, Lin L, Wang HJ, et al. Suppression of early experimental osteoarthritis by in vivo delivery of the adenoviral vector-mediated NF-kappaBp65-specific siRNA. Osteoarthritis Cartilage. 2008; 16(2):174-184.

10. Hsieh JL, Shen PC, Shiau AL, et al. Intraarticular gene transfer of thrombospondin-1 suppresses the disease progression of experimental osteoarthritis. J Orthop Res. 2010;28(10):1300-1306.

11. Hsieh JL, Shen PC, Shiau AL, et al. Adenovirus-mediated kallistatin gene transfer ameliorates disease progression in a rat model of osteoarthritis induced by anterior cruciate ligament transection. Hum Gene Ther. 2009;20(2):147-158.

12. Shen PC, Shiau AL, Jou IM, et al. Inhibition of cartilage damage by pro-opiomelanocortin prohormone overexpression in a rat model of osteoarthritis. Exp Biol Med (Maywood). 2011;236(3):334-340.

13. Oh H, Chun $\mathrm{CH}$, Chun JS. Dkk-1 expression in chondrocytes inhibits experimental osteoarthritic cartilage destruction in mice. Arthritis Rheum. 2012;64(8):2568-2578.
14. Santangelo KS, Nuovo GJ, Bertone AL. In vivo reduction or blockade of interleukin-1beta in primary osteoarthritis influences expression of mediators implicated in pathogenesis. Osteoarthritis Cartilage. 2012; 20(12):1610-1618.

15. Nixon AJ, Goodrich LR, Scimeca MS, et al. Gene therapy in musculoskeletal repair. Ann N Y Acad Sci. 2007;1117:310-327.

16. Weimer A, Madry H, Venkatesan JK, et al. Benefits of recombinant adeno-associated virus (rAAV)-mediated insulin-like growth factor I (IGF-I) overexpression for the long-term reconstruction of human osteoarthritic cartilage by modulation of the IGF-I axis. $\mathrm{Mol}$ Med. 2012;18:346-358.

17. Cucchiarini M, Terwilliger EF, Kohn D, Madry H. Remodeling of human osteoarthritic cartilage by FGF-2, alone or combined with Sox 9 via rAAV gene transfer. J Cell Mol Med. 2009;13(8B):2476-2488.

18. Matsumoto T, Cooper GM, Gharaibeh B, et al. Cartilage repair in a rat model of osteoarthritis through intraarticular transplantation of muscle-derived stem cells expressing bone morphogenetic protein 4 and soluble Flt-1. Arthritis Rheum. 2009;60(5):1390-1405.

19. Cucchiarini M, Thurn T, Weimer A, Kohn D, Terwilliger EF, Madry H. Restoration of the extracellular matrix in human osteoarthritic articular cartilage by overexpression of the transcription factor SOX9. Arthritis Rheum. 2007;56(1):158-167.

20. Ruan MZ, Erez A, Guse K, et al. Proteoglycan 4 expression protects against the development of osteoarthritis. Sci Transl Med. 2013; 5(176):176ra134.

21. O'Keefe RJ, Puzas JE, Brand JS, Rosier RN. Effect of transforming growth factor-beta on DNA synthesis by growth plate chondrocytes: modulation by factors present in serum. Calcif Tissue Int. 1988;43(6): $352-358$.

22. Redini F, Galera P, Mauviel A, Loyau G, Pujol JP. Transforming growth factor beta stimulates collagen and glycosaminoglycan biosynthesis in cultured rabbit articular chondrocytes. FEBS Lett. 1988; 234(1):172-176.

23. van Beuningen HM, van der Kraan PM, Arntz OJ, van den Berg WB. Protection from interleukin 1 induced destruction of articular cartilage by transforming growth factor beta: studies in anatomically intact cartilage in vitro and in vivo. Ann Rheum Dis. 1993;52(3):185-191.

24. Shi S, Mercer S, Eckert GJ, Trippel SB. Regulation of articular chondrocyte aggrecan and collagen gene expression by multiple growth factor gene transfer. J Orthop Res. 2012;30(7):1026-1031.

25. Shi S, Mercer S, Eckert GJ, Trippel SB. Growth factor transgenes interactively regulate articular chondrocytes. J Cell Biochem. 2013; 114(4):908-919.

26. Shuler FD, Georgescu HI, Niyibizi C, et al. Increased matrix synthesis following adenoviral transfer of a transforming growth factor beta1 gene into articular chondrocytes. J Orthop Res. 2000;18(4):585-592.

27. Hao J, Yao Y, Varshney RR, et al. Gene transfer and living release of transforming growth factor-beta3 for cartilage tissue engineering applications. Tissue Eng Part C Methods. 2008;14(4):273-280.

28. Noh MJ, Copeland RO, Yi Y, et al. Pre-clinical studies of retrovirally transduced human chondrocytes expressing transforming growth factorbeta-1 (TG-C). Cytotherapy. 2010;12(3):384-393.

29. Madry H, Cucchiarini M, Terwilliger EF, Trippel SB. Recombinant adeno-associated virus vectors efficiently and persistently transduce chondrocytes in normal and osteoarthritic human articular cartilage. Hum Gene Ther. 2003;14(4):393-402.

30. Cucchiarini M. Human gene therapy: novel approaches to improve the current gene delivery systems. Discov Med. 2016;21(118):495-506.

31. Ulrich-Vinther M, Stengaard C, Schwarz EM, Goldring MB, Soballe K. Adeno-associated vector mediated gene transfer of transforming growth factor-beta1 to normal and osteoarthritic human chondrocytes stimulates cartilage anabolism. Eur Cell Mater. 2005;10:40-50.

32. Venkatesan JK, Rey-Rico A, Schmitt G, Wezel A, Madry H, Cucchiarini M. rAAV-mediated overexpression of TGF-beta stably restructures human osteoarthritic articular cartilage in situ. J Transl Med. 2013;11:211. 
33. Cottard V, Valvason C, Falgarone G, Lutomski D, Boissier MC, Bessis N. Immune response against gene therapy vectors: influence of synovial fluid on adeno-associated virus mediated gene transfer to chondrocytes. J Clin Immunol. 2004;24(2):162-169.

34. Rey-Rico A, Cucchiarini M. Controlled release strategies for rAAVmediated gene delivery. Acta Biomater. 2016;29:1-10.

35. Rey-Rico A, Cucchiarini M. Recent tissue engineering-based advances for effective rAAV-mediated gene transfer in the musculoskeletal system. Bioengineered. 2016;7(3):175-188.

36. Rey-Rico A, Venkatesan JK, Frisch J, et al. PEO-PPO-PEO micelles as effective rAAV-mediated gene delivery systems to target human mesenchymal stem cells without altering their differentiation potency. Acta Biomater. 2015;27:42-52.

37. Rey-Rico A, Frisch J, Venkatesan JK, et al. PEO-PPO-PEO carriers for rAAV-mediated transduction of human articular chondrocytes in vitro and in a human osteochondral defect model. ACS Appl Mater Interfaces. 2016;8(32):20600-20613.

38. Samulski RJ, Chang LS, Shenk T. Helper-free stocks of recombinant adeno-associated viruses: normal integration does not require viral gene expression. J Virol. 1989;63(9):3822-3828.

39. Samulski RJ, Chang LS, Shenk T. A recombinant plasmid from which an infectious adeno-associated virus genome can be excised in vitro and its use to study viral replication. J Virol. 1987;61(10):3096-3101.
40. Frisch J, Venkatesan JK, Rey-Rico A, Schmitt G, Madry H, Cucchiarini M Determination of the chondrogenic differentiation processes in human bone marrow-derived mesenchymal stem cells genetically modified to overexpress transforming growth factor-beta via recombinant adenoassociated viral vectors. Hum Gene Ther. 2014;25(12):1050-1060.

41. Bartlett JS, Wilcher R, Samulski RJ. Infectious entry pathway of adenoassociated virus and adeno-associated virus vectors. J Virol. 2000; 74(6):2777-2785.

42. Elsler S, Schetting S, Schmitt G, Kohn D, Madry H, Cucchiarini M. Effective, safe nonviral gene transfer to preserve the chondrogenic differentiation potential of human mesenchymal stem cells. J Gene Med. 2012;14(7):501-511.

43. Stanton LA, Sabari S, Sampaio AV, Underhill TM, Beier F. p38 MAP kinase signaling is required for hypertrophic chondrocyte differentiation. Biochem J. 2004;378(Pt 1):53-62.

44. Woods A, Wang G, Beier F. RhoA/ROCK signaling regulates Sox 9 expression and actin organization during chondrogenesis. J Biol Chem. 2005;280(12):11626-11634.

45. Calcedo R, Wilson JM. Humoral immune response to AAV. Front Immunol. 2013;4:341.

46. Poole AR, Kojima T, Yasuda T, Mwale F, Kobayashi M, Laverty S. Composition and structure of articular cartilage: a template for tissue repair. Clin Orthop Relat Res. 2001;(391 Supp1):S26-S33. 


\section{Supplementary material}

\section{PF68/rAAV-Cy3-hTGF- $\beta$}

Day 1

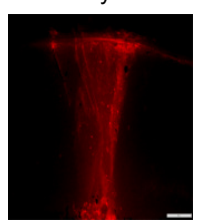

Day 2

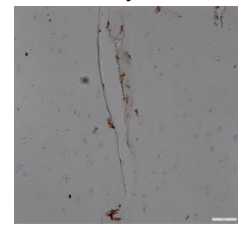

Day 2

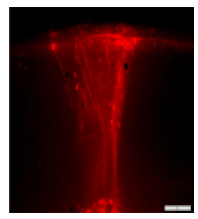

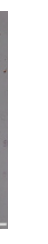

rAAV-Cy3-hTGF- $\beta$

Day 1

Day 2

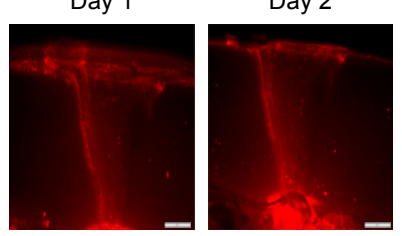

Day 2

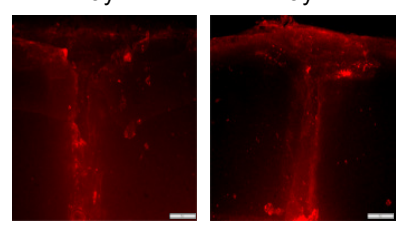

Day 2
No vector

Day 1

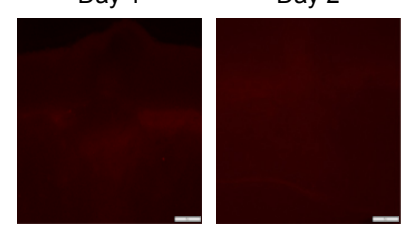

Day 2
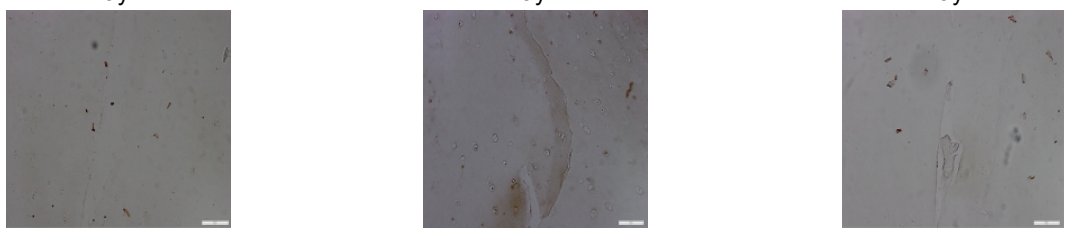

Figure SI Characterization of rAAV-hTGF- $\beta$-mediated gene transfer in human osteochondral defect cultures via polymeric micelles.

Notes: Defects were prepared, incubated with Cy3-rAAV/polymeric micelles and processed after 2 days to monitor the diffusion and bioactivity of the vectors by analysis of live fluorescence and immunohistochemical detection, respectively (magnification $\times 2$ ) in cross-sections from the defects as described in the "Cy3 labeling" and "Histological and immunocyto/histochemical analyses" sections. Scale bar represents $1 \mathrm{~mm}$.

Abbreviations: hTGF, human transforming growth factor; rAAV, recombinant adeno-associated virus.

\section{Publish your work in this journal}

The International Journal of Nanomedicine is an international, peerreviewed journal focusing on the application of nanotechnology in diagnostics, therapeutics, and drug delivery systems throughout the biomedical field. This journal is indexed on PubMed Central, MedLine, CAS, SciSearch ${ }^{\circledR}$, Current Contents ${ }^{\circledR} /$ Clinical Medicine,
Journal Citation Reports/Science Edition, EMBase, Scopus and the Elsevier Bibliographic databases. The manuscript management system is completely online and includes a very quick and fair peer-review system, which is all easy to use. Visit http://www.dovepress.com/ testimonials.php to read real quotes from published authors. 